\title{
Brain functional changes across the different phases of bipolar disorder
}

Edith Pomarol-Clotet, Silvia Alonso-Lana, Noemi Moro, Salvador Sarró, Mar C. Bonnin, José M. Goikolea, Paloma Fernández-Corcuera, Benedikt L. Amann, Anna Romaguera, Eduard Vieta, Josep Blanch, Peter J. McKenna and Raymond Salvador

\section{Background}

Little is known about how functional imaging changes in bipolar disorder relate to different phases of the illness.

\begin{abstract}
Aims
To compare cognitive task activation in participants with bipolar disorder examined in different phases of illness.
\end{abstract}

\section{Method}

Participants with bipolar disorder in mania $(n=38)$, depression $(n=38)$ and euthymia $(n=38)$, as well as healthy controls $(n=38)$, underwent functional magnetic resonance imaging during performance of the n-back working memory task. Activations and de-activations were compared between the bipolar subgroups and the controls, and among the bipolar subgroups. All participants were also entered into a linear mixed-effects model.

\section{Results}

Compared with the controls, the mania and depression subgroups, but not the euthymia subgroup, showed reduced activation in the dorsolateral prefrontal cortex, the parietal cortex and other areas. Compared with the euthymia subgroup, the mania and depression subgroups showed hypoactivation in the parietal cortex. All three bipolar subgroups showed failure of de-activation in the ventromedial frontal cortex. Linear mixed-effects modelling revealed a further cluster of reduced activation in the left dorsolateral prefrontal cortex in the patients; this was significantly more marked in the mania than in the euthymia subgroup

\section{Conclusions}

Bipolar disorder is characterised by mood state-dependent hypoactivation in the parietal cortex. Reduced dorsolateral prefrontal activation is a further feature of mania and depression, which may improve partially in euthymia. Failure of de-activation in the medial frontal cortex shows trait-like characteristics.

\section{Declaration of interest}

None.
Functional imaging studies in bipolar disorder have had heterogeneous and at times confusing findings, although there is now a broad consensus that the disorder is characterised by overactivity in subcortical structures such as the amygdala, hippocampus and basal ganglia, coupled with reduced activity in prefrontal and some other cortical regions. ${ }^{1-4}$ Recent meta-analyses suggest that this pattern is seen both at rest and in studies using task activation, ${ }^{5}$ although the pattern differs to some extent depending on whether cognitive or emotional tasks (typically facial emotion processing) are used. ${ }^{6}$ What remains less clear is the 'state' $v$. 'trait' characteristics of functional imaging abnormality in bipolar disorder, i.e. whether there are differences between patients in manic and depressed episodes and to what extent changes seen in both phases of illness persist into euthymia.

In Kupferschmidt \& Zakzanis' ${ }^{5}$ meta-analysis of 55 studies that pooled conventional effect-size data, resting and task-related changes were seen in episodes of illness and in euthymia but the differences between phases were complex. Cortical hypoactivity and limbic hyperactivity was found to be greater in patients in a manic phase than in euthymia, and patients in a depressed phase showed greater hypoactivation in frontal regions than in euthymia. However, patients in euthymia showed more evidence of limbic hyperactivity than those with depression. Abnormalities were also more pronounced in patients in a manic than in depressed phase. Rather differently, Chen et al's ${ }^{6}$ meta-analysis of voxel-based functional magnetic resonance imaging (fMRI) studies found that changes (reduced activation) were restricted to the lingual gyrus in 26 studies carried out on participants in a euthymic phase. There were only relatively few studies carried out exclusively on participants in manic and depressed phases
(8 and 7 respectively), and so their findings - decreased activation in the inferior frontal gyrus in mania and no change in depression - may not have been reliable.

In what appears to be the only contemporary study to directly compare patients across phases, Townsend et al ${ }^{7}$ examined 13 patients in a manic phase, 14 in a depressed phase and 15 in a euthymic phase, as well as 14 healthy controls, during performance of the n-back working memory task. Citing a lack of availability of methods for analysing differences among four groups at the whole-brain level, the authors only examined two predetermined regions of interest (ROIs), the left and right dorsolateral prefrontal cortex and the left and right posterior parietal cortex. They found reduced activation in both ROIs in mania, depression and euthymia, with no significant variation across phase.

Over the past decade the importance not only of task-related activations but also de-activations has become increasingly recognised. This follows the discovery of the so-called default mode network, an interconnected set of brain regions that are highly active at rest but de-activate during performance of a wide range of attention- demanding tasks. ${ }^{8,9}$ Prominent among these regions are two midline cortical areas, the medial frontal cortex anteriorly and the posterior cingulate cortex/precuneus posteriorly. Recent evidence suggests that bipolar disorder may also be characterised by de-activation changes in the default mode network. Thus, Pomarol-Clotet et al ${ }^{10}$ found failure of de-activation in the ventromedial frontal cortex during performance of the n-back task in participants in a manic episode compared with healthy controls, and Fernández-Corcuera et $\mathrm{al}^{11}$ had similar findings in patients with bipolar depression. 
Strakowski et al, ${ }^{12}$ in contrast, found significantly greater de-activation in participants with first-episode mania compared with healthy controls in the bilateral posterior cingulate cortex. So far there has been only one study of patients in the euthymic phase: Allin et $a l^{13}$ found no changes in the medial frontal or lateral parietal nodes of the default mode network during performance of a paced verbal fluency task, but failure of de-activation was seen in the retrosplenial cortex and adjacent precuneate cortex, an area conforming reasonably closely to the posterior midline node.

The aim in this study was to examine whether task-related activations differed in patients in manic, depressed and euthymic bipolar phases, and also to further clarify the pattern of de-activation changes associated with the disorder. We used a cognitive task, the n-back working memory task, which has been found to be associated with functional imaging changes in both illness phases of bipolar disorder ${ }^{7,10,11}$ and in euthymia (for a review see Cremaschi et ll $^{14}$ ), and which we have found to reliably produce de-activation in the territory of the default mode network..$^{10,11,15,16}$ We used whole-brain voxel-based analysis and were able to take advantage of advances in fMRI methodology to carry out a conjoint analysis of the four groups of participants (i.e. the three bipolar disorder subgroups and the healthy control group).

\section{Method}

\section{Participants}

The patient sample was recruited from three hospitals in Barcelona, Benito Menni CASM, Hospital Clínic and Hospital General de Granollers. All patients met DSM-IV ${ }^{17}$ criteria for bipolar disorder, made up of three subgroups, mania ( $n=38$, all type I), depression ( $n=38,32$ type I and 6 type II) and euthymia $(n=38$, all type I). The participants in the mania subgroup were required to have a Young Mania Rating Scale (YMRS) ${ }^{18}$ score $\geqslant 18$ and those in the depression subgroup to have a score of $\geqslant 15$ on the Hamilton Rating Scale for Depression (21-items, HRSD). ${ }^{19}$ Participants in the euthymia subgroup were required to have had no episodes of illness for at least 3 months and a score on the HRDS- 21 of $\leqslant 8$ and YMRS of $\leqslant 8$ at the time of scanning. All patients were right handed.

Patients were excluded if: (a) they were younger than 18 or older than 65 years, (b) they had a history of brain trauma or neurological disease, or (c) there was alcohol/substance misuse in the 12 months prior to participation. Patients who had undergone electroconvulsive therapy in the previous 12 months were also excluded.

In the mania subgroup, patients were taking the following medications: mood stabilisers (lithium $n=20$; other mood stabilisers $n=5)$; antidepressants $(n=2)$ and antipsychotics $(n=28$, second generation 21; first generation 2; combination 5); all medication data were missing for 1 patient. In the depression subgroup, patients were taking mood stabilisers (lithium $n=25$; other mood stabilisers $n=9)$ and/or antidepressants $(n=22)$ and antipsychotics ( $n=20$, all second generation). Most of the participants in the euthymia subgroup were on mood stabilisers (lithium $n=28$; other mood stabilisers $n=8$ ); some patients were taking antidepressants $(n=8)$ and some were also taking antipsychotics ( $n=21$, all second generation).

A healthy control group $(n=38)$ was recruited via poster and web-based advertisement in the hospital and local community, plus word-of-mouth requests from staff in the research unit. They met the same exclusion criteria as the bipolar group. They were interviewed and excluded if they reported a history of mental illness and/or treatment with psychotropic medication other than non-regular use of benzodiazepines or similar drugs for insomnia. They were also questioned about family history of mental illness and excluded if a first-degree relative had experienced symptoms consistent with major psychiatric disorder and/or had received any form of in- or out-patient psychiatric care. All were right handed.

All four groups were matched for age, gender and IQ, as estimated by the Word Accentuation Test (Test de Acentuación de Palabras, TAP), ${ }^{20}$ a test requiring pronunciation of Spanish words whose accents have been removed. The TAP has been standardised against the Wechsler Adult Intelligence Scale

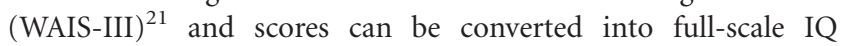
estimates. $^{22}$ Both the bipolar and control groups were also required to have a current IQ in the normal range (i.e. $\geqslant 70$ ), as measured using four subtests of the WAIS-III: vocabulary, similarities, block design and matrix reasoning.

All participants gave written informed consent. The study was approved by the local research ethics committees.

\section{Scanning procedure}

While being scanned, individuals performed a sequential-letter version of the n-back task ${ }^{23}$ in the scanner (Fig. 1). Two levels of memory load (1-back and 2-back) were presented in a blocked design manner. Each block consisted of 24 letters that were shown every $2 \mathrm{~s}$ ( $1 \mathrm{~s}$ on, $1 \mathrm{~s}$ off) and all blocks contained five repetitions (1-back and 2-back depending on the block) located randomly within the blocks. Individuals had to indicate repetitions by pressing a button. Four 1-back and four 2-back blocks were presented in an interleaved way, and between them a baseline stimulus (an asterisk flashing with the same frequency as the letters) was presented for $16 \mathrm{~s}$. To identify which task had to be performed, characters were shown in green in 1-back blocks and in red in 2-back blocks. All participants first went through a training session outside the scanner.

Task performance was measured using the signal detection theory index of sensitivity (d') of ability to discriminate targets from non-targets. ${ }^{24}$ Higher values of $d$ ' indicate better ability to discriminate between targets and distractors. Participants who had negative d' values in either or both of the 1-back and 2-back versions of the task, which suggests that they were not performing it, were a priori excluded from the study.

In each individual scanning session 266 volumes were acquired from a 1.5-T GE Signa scanner. A gradient-echo echoplanar imaging (EPI) sequence depicting the blood oxygenation level-dependent (BOLD) contrast was used. Each volume contained 16 axial planes acquired with the following parameters: repetition time $(\mathrm{TR})=2000 \mathrm{~ms}$, echo time $(\mathrm{TE})=20 \mathrm{~ms}$, flip angle $70^{\circ}$, section thickness, $7 \mathrm{~mm}$, section skip, $0.7 \mathrm{~mm}$, in-plane resolution, $3 \times 3 \mathrm{~mm}$. The first 10 volumes were discarded to avoid $T_{1}$ saturation effects.

Individual fMRI analyses were performed with the FEAT module, included in FSL software (version 4.19 for Linux) ${ }^{25}$ In the preprocessing phase, images were corrected for movement, co-registered and spatially filtered with a Gaussian filter (fullwidth at half maximum $(F W H M)=5 \mathrm{~mm}$ ). To minimise unwanted movement-related effects, individuals with an estimated maximum absolute movement $>3.0 \mathrm{~mm}$ or an average absolute movement $>0.3 \mathrm{~mm}$ were excluded from the study. General linear models were fitted to generate individual activation maps for the contrast comparing blocks of baseline with blocks of the 2-back level of the task. To further reduce the potential effect of movement, values of movement parameters were included as nuisance covariates in the fitting of individual linear models. 

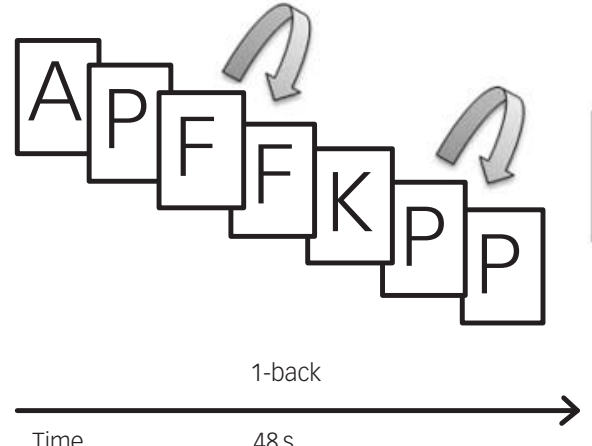

$48 \mathrm{~s}$
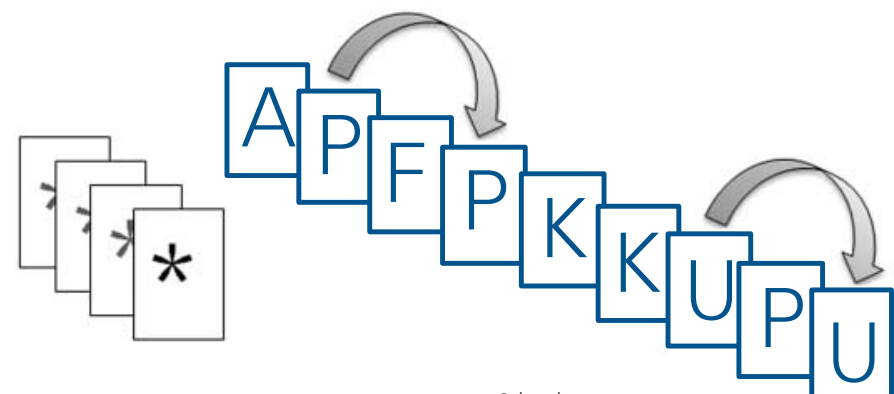

2-back

$48 s$

Fig. 1 Sequential-letter version of the n-back task with two levels of memory load, 1-back (black) and 2-back (blue).

Before the group analyses, images were normalised to a common stereotaxic space (Montreal Neurologic Institute (MNI) template).

\section{Group comparisons}

The same FEAT module was used to fit a linear mixed-effects model including the baseline $v$. 2-back activation images for the four independent groups. Using this model, each bipolar subgroup was individually compared with the healthy controls. The bipolar subgroups were also compared with each other, i.e. mania $v$. euthymia, depression $v$. euthymia and mania $v$. depression. Statistical tests on these contrasts were carried out at the cluster level with a family-wise corrected $P$-value of 0.05 using Gaussian random field methods. The default threshold of $z=2.3$ was used to define the initial set of clusters.

Additionally, an ANOVA was run on all four groups together, using the same fitted linear mixed-effects model that defined clusters of difference between any of the four groups. Significant clusters were then taken as ROIs and were used to draw exploratory boxplots visualising the relative levels of activation/ de-activation for the four groups in areas of functional abnormality.

\section{Results}

Demographic and clinical data on the bipolar and control groups are shown in Table 1. The groups were well-matched for age, gender and TAP-estimated IQ. However, the healthy control group had a higher mean current IQ than the bipolar subgroups, which was significant in the case of the mania and depression subgroups. The three bipolar subgroups did not differ significantly in duration of illness.

\section{Performance on the n-back test}

There were significant differences among the groups on both the 1 -back version and the 2-back versions of the test $(F=6.66$, $P<0.001$ and $F=12.74, P<0.001$, respectively). In the 1-back version, post hoc testing (Tukey HSD) indicated that the mania subgroup performed significantly more poorly than the control group (mean d' 3.36 (s.d.=1.10) v. 4.34 (s.d.=0.76), <0.001) as did the depression subgroup (mean d' 3.75 (s.d.=1.17), $P=0.04$ ); however, the euthymia subgroup did not perform significantly differently from the control group (mean d' 3.91 (s.d. $=1.11), P<0.23$ ). Results were similar in the 2-back version, with the mania subgroup performing significantly worse than the control group (mean d' 2.02 (s.d.=1.05) v. 3.22 (s.d.=0.86), $P<0.001$ ), and the depression subgroup performing worse than the control group (mean d' 2.28 (s.d. $=1.13$ ), $P<0.001$ ); however, the performance of the euthymia subgroup did not differ from the control group (mean d' 2.77 (s.d. $=0.90$ ), $P<0.15$ ). Within the bipolar subgroups the only significant difference was between the mania and the euthymia subgroups $(P=0.006)$.

\section{Neuroimaging findings}

As in previous studies by our group, ${ }^{10,11,15}$ the 2-back $v$. baseline contrast delivered larger and more extensive activations and de-activations than the 1-back $v$. baseline contrast. Therefore, in what follows only the results for this contrast are reported.

Maps of mean activations for the three bipolar subgroups and the control group in the 2-back $v$. baseline contrast are shown in online Fig. DS1. Briefly, at $P<0.05$ corrected, the healthy control group showed a pattern of bilateral activations in the anterior insula, the dorsolateral prefrontal cortex, and the precentral gyri, supplementary motor areas, cerebellum, thalamus, basal ganglia, and parts of the temporal and parietal cortex. They also showed de-activations: these were seen bilaterally in the medial frontal cortex, the amygdala, the hippocampus and adjacent cortical regions, the medial parietal cortex extending to primary visual areas, the posterior insula and the lateral parietal cortex.

Activations and de-activations in the mania, depression and euthymia subgroups followed a broadly similar pattern to that seen in the control group. However, the clusters were noticeably less extensive and less highly activated/de-activated, particularly in the mania and depression subgroups.

\section{Comparisons between the individual subgroups and the healthy control group}

Mania subgroup $v$. control group. As shown in Figure 2, at $P<0.05$ corrected, the mania subgroup showed significant failure of activation in bilateral clusters involving the dorsolateral prefrontal cortex, the parietal cortex and the precuneus (left: 12272 voxels, peak activation in Brodmann area (BA) 6/8, MNI $(-30,-2,62), \quad z$-score $=5.06, \quad P=7.13 \times 10^{-17}$; right: 1477 voxels, peak activation in BA $6 / 8, \mathrm{MNI}(24,6,58), z$-score $=5.29$, $P=0.002)$. Clusters of reduced activation were also seen in the basal ganglia and thalamus bilaterally (right: 1409 voxels, peak activation in MNI $(18,8,16), z$-score $=4.1, P=0.002)$; left: 1208 voxels, peak activation in MNI $(-18,-10,18), z$-score $=3.87$, $P=0.006)$ and the cerebellum (right: 1654 voxels, peak activation in MNI $(30,-44,-32), z$-score $=4.21, P=0.0008$; left: 1259 voxels, peak activation in MNI $(-44,-70,-10), z$-score $=3.87$, $P=0.005)$.

The mania subgroup also showed failure of de-activation in the ventromedial prefrontal cortex relative to the control group (2605 voxels, peak activation in $\mathrm{MNI}(-2,46,-28), z$-score $=4.73$, $\left.P=1.87 \times 10^{-5}\right)$. 


\begin{tabular}{|c|c|c|c|c|c|c|c|c|c|}
\hline & $\begin{array}{c}\text { Mania } \\
\text { subgroup } \\
(n=38)\end{array}$ & $\begin{array}{l}\text { Depression } \\
\text { subgroup } \\
(n=38)\end{array}$ & $\begin{array}{c}\text { Euthymia } \\
\text { subgroup } \\
(n=38)\end{array}$ & $\begin{array}{l}\text { Control } \\
\text { group } \\
(n=38)\end{array}$ & $F$ & $\chi^{2}$ & $K W$ & $P$ & $\begin{array}{l}\text { Post } \\
\text { hoc }\end{array}$ \\
\hline Age, years: mean (s.d.) & $39.74(11.36)$ & $39.89(10.39)$ & $40.00(8.78)$ & $39.68(8.88)$ & 0.01 & & & 0.99 & \\
\hline Gender, men/women: $n$ & $18 / 20$ & $17 / 21$ & $17 / 21$ & $18 / 20$ & & 0.11 & & 0.99 & \\
\hline TAP, mean (s.d.) & $22.75(4.52)$ & $22.47(4.85)$ & $23.67(3.15)$ & $22.71(3.28)$ & 0.62 & & & 0.60 & \\
\hline TAP_FISQ, mean (s.d.) & $101.81(8.65)$ & $101.33(9.39)$ & $103.50(6.02)$ & $101.66(6.21)$ & 0.57 & & & 0.63 & \\
\hline WAIS-III, mean (s.d.) & $95.15(14.28)$ & $93.17(15.05)$ & $97.47(13.87)$ & $105.61(14.59)$ & 5.06 & & & 0.002 & $\mathrm{M}, \mathrm{D}<\mathrm{C}$ \\
\hline $\begin{array}{l}\text { Duration of illness, years: } \\
\text { mean (s.d.) }\end{array}$ & $11.59(11.67)$ & $14.51(10.17)$ & $13.03(7.28)$ & N/A & 0.81 & & & 0.45 & \\
\hline YMRS score, mean (s.d.) & $21.84(3.67)$ & $1.29(2.24)$ & $1.41(1.88)$ & $\mathrm{N} / \mathrm{A}$ & 717.81 & & & $<0.001$ & $D, E<M$ \\
\hline HRSD score, mean (s.d.) & $4.14(3.65)$ & $22.13(4.03)$ & $2.73(2.18)$ & $\mathrm{N} / \mathrm{A}$ & 383.07 & & & $<0.001$ & $M, E<D$ \\
\hline CGI score mean (s.d.) & $4.66(0.72)$ & $4.51(0.65)$ & $2.06(1.03)$ & N/A & & & 60.39 & $<0.001$ & $M, D>E$ \\
\hline GAF score, mean (s.d.) & $45.81(11.05)$ & $44.77(11.90)$ & 76.78 (11.17) & N/A & 84.43 & & & $<0.001$ & $M, D<E$ \\
\hline History of psychosis, yes/no: $n$ & $30 / 8$ & $24 / 10^{*}$ & $30 / 8$ & N/A & & 1.31 & & 0.52 & \\
\hline
\end{tabular}

Depression subgroup $v$. control group. Once again the patients showed a pattern of hypo-activation in the bilateral dorsolateral prefrontal cortex extending to the basal ganglia and thalamus (4415 voxels, peak activation in MNI $(-32,-2,50), z$-score $=4.71$, $\left.P=1.9 \times 10^{-14}\right)$; in the bilateral parietal cortex and precuneus (2687 voxels, peak activation in MNI $(8,-64,62), z$-score $=4.86$, $\left.P=1.3 \times 10^{-5}\right)$; and in the cerebellum (2707 voxels, peak activation in MNI $(-32,-54,-30), z$-score $\left.=4.89, P=1.2 \times 10^{-5}\right)$. Like the mania subgroup, the depression subgroup also showed failure of de-activation in the ventromedial prefrontal cortex, (4415 voxels, peak activation in MNI $(0,46,-20), z$-score $\left.=4.68, P=5.9 \times 10^{-8}\right)$ (Fig. 2).

Euthymic subgroup $v$. control group. In contrast to the other two groups, the euthymia subgroup did not show any clusters of reduced activation compared with the control group. However,

(a)

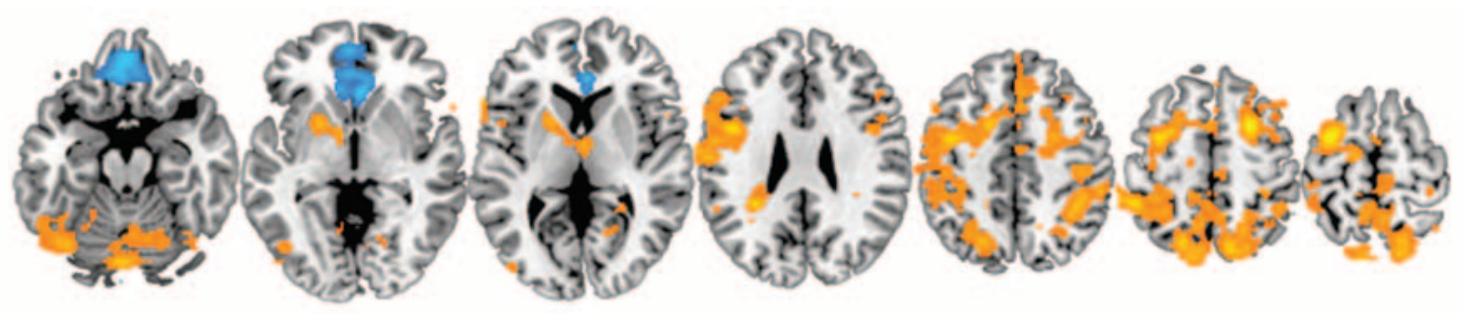

(b)

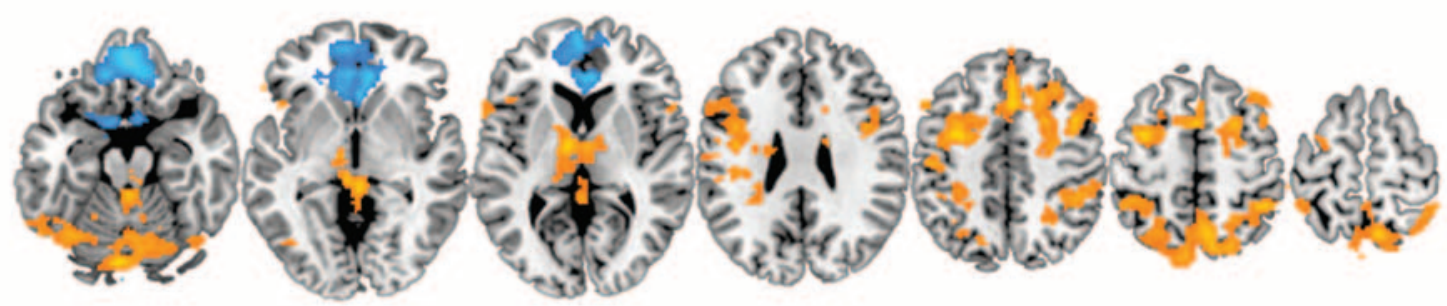

(c)

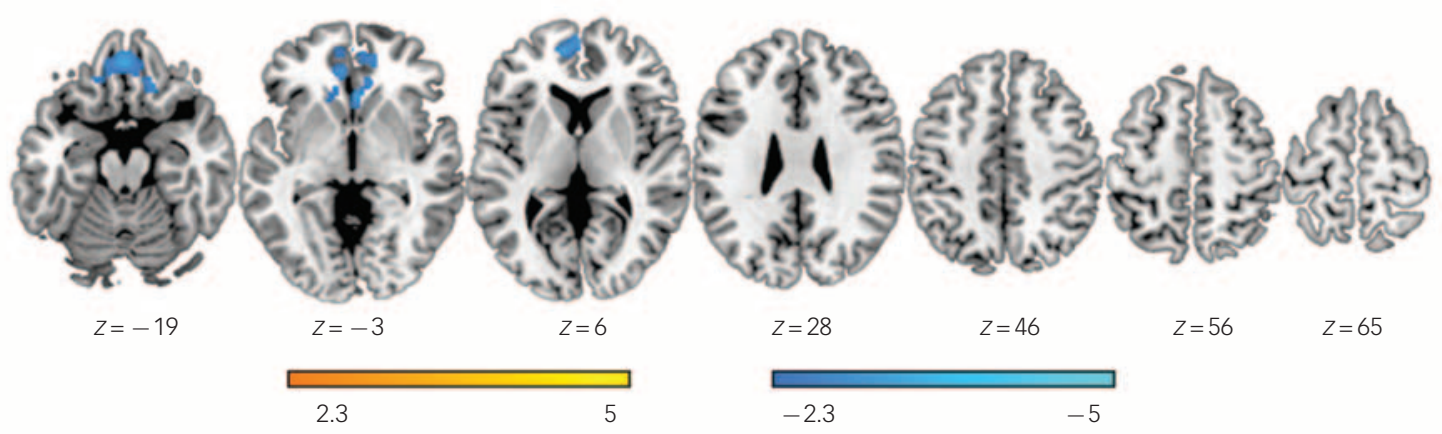

Fig. 2 Brain clusters showing statistically significant differences in the 2-back $v$. baseline contrast (at $P<0.05$ corrected) among the three bipolar subgroups compared with the controls.

(a) Mania subgroup $v$. control group; (b) depression subgroup $v$. control group; and (c) euthymia subgroup $v$. control group. The right side of the image is the right side of the brain 
failure of de-activation was again seen in the ventromedial frontal cortex (3511 voxels, with peak activation in BA 25 , MNI $(-6,16$, $-10), z$-score $\left.=4.12, P=1.31 \times 10^{-6}\right)($ Fig. 2$)$.

Since the mania and depression subgroups showed significantly worse n-back task performance than the healthy control group, we repeated these comparisons including performance as a covariate. Although there was a reduction in the area of some of the clusters found, for the most part they were still evident in the same locations. The findings are shown in online Fig. DS2.

\section{Comparisons within the bipolar subgroups}

Mania $v$. euthymia subgroup. The mania subgroup showed two clusters of significantly reduced activation compared with the euthymia subgroup. These were symmetrically located in the left and right dorsal parietal cortex and precuneus. The cluster on the left had a larger extension (2229 voxels, peak activation in BA 40, MNI $(-46,-42,56), z$-score $\left.=3.93, P=7.8310^{-5}\right)$, than that on the right (1368 voxels, peak activation in BA 7, MNI (26, $-50,56), z$-score $\left.=3.9, P=2.97 \times 10^{-3}\right)$. The findings are shown in Fig. 3.

Depression $v$. euthymia subgroup. Results for this comparison were similar to those for the mania $v$. euthymia contrast. Once again the depression subgroup showed significantly reduced activation in the dorsal parietal cortex and precuneus. This time, however, the reduction was unilateral, being seen only on the right (982 voxels, peak activation in BA 40, MNI $(40,-44,56)$, $z$-score $=3.95, P=0.02)$. The findings are also shown in Fig. 3 .

Mania $v$. depression subgroup. No significant differences in levels of activation or de-activation were found between these two phases of illness. For the comparison between the mania and euthymia subgroups, the only pair-wise comparison where the two groups differed significantly in $\mathrm{n}$-back performance, the analysis was repeated adding $d$ ' as a covariate. The results remained similar. The findings are shown in online Fig. DS2.

\section{Four-group ANOVA and ROI analysis}

Results from the ANOVA including the three bipolar subgroups and the control group are shown in Fig. 4. Four clusters of significant difference between at least one group and the others were found. Two of the clusters were symmetrically located in the left and right dorsal parietal cortex, similar to the significant clusters found in the mania $v$. euthymia and in the depression $v$. euthymia contrasts (right: 1582 voxels, peak activation in MNI (40, $-46,56), z$-score $=4.05, P=0.001$; left 1118 voxels, peak activation in MNI $(-58,-40,54), z$-score $=4.17$, $P=0.009$ ). Boxplots of mean activations in ROIs based on these two clusters indicated significantly reduced levels of activation in both phases of illness compared with euthymia and the control group. However, the euthymia subgroup did not differ significantly from the control group in either of the clusters (see online Table DS1 for details of the means, standard deviations and significance levels).

A third cluster, also found in the individual analyses, was located in the ventromedial frontal cortex (1943 voxels, peak activation in MNI $(-2,46,-28), z$-score $=5.06, P=0.0002)$. The accompanying boxplots indicate significantly lower for the mania, depression and euthymia subgroups than the de-activation control group.

This analysis also produced a new cluster. This was located in the left dorsolateral prefrontal cortex (1256 voxels, peak activation in BA 6, MNI $(-34,-2,54), z$-score $=4.32, P=0.005)$. As can be seen from the boxplots, there was significantly reduced activation in the mania, depression and euthymia subgroups compared with the control group. The mania subgroup also showed significantly reduced activation compared with the euthymia subgroup.

\section{Discussion}

\section{Main findings}

The analyses carried out in this study provide evidence that bipolar disorder is characterised by both mood-state-dependent and mood-state-independent functional imaging abnormalities.

(a)

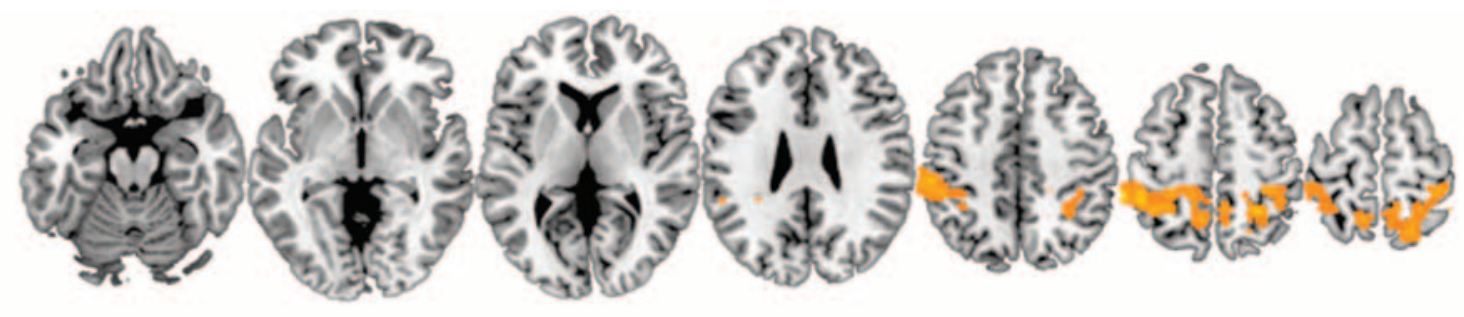

(b)

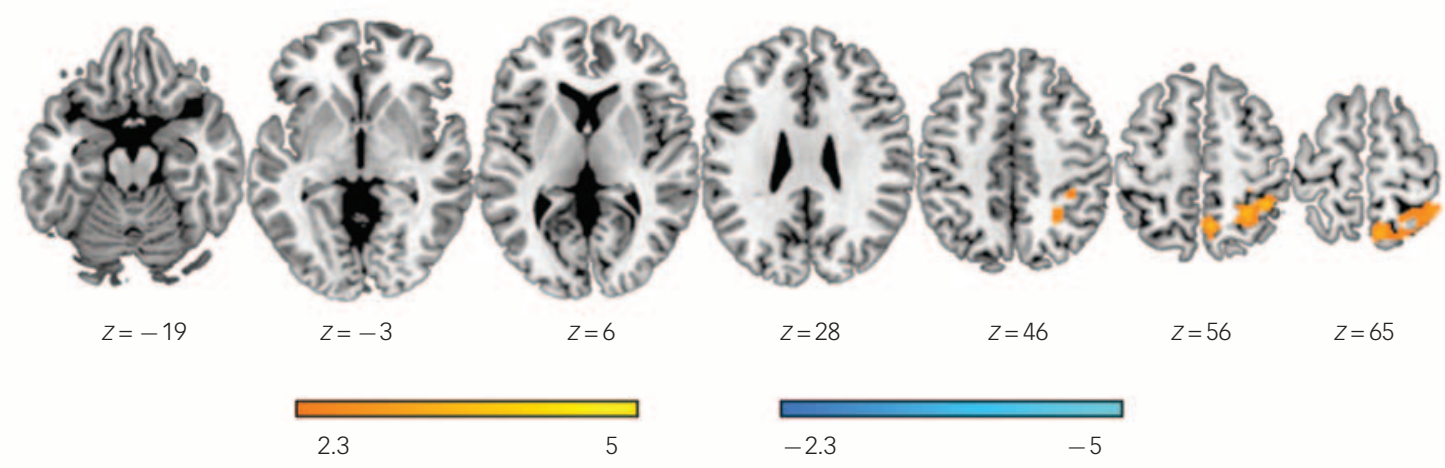

Fig. 3 Brain clusters showing statistically significant differences in the 2-back $\boldsymbol{v}$. baseline contrast (at $P<0.05$ corrected) in the mania and depression subgroups compared with the euthymia subgroup.

(a) Mania $v$. euthymia subgroup and (b) depression $v$. euthymia subgroup. No differences were found when the mania subgroup were compared with the depression subgroup. The right side of the image is the right side of the brain. 
(a)

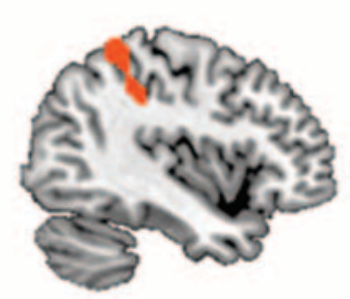

$x=-41$

(b)

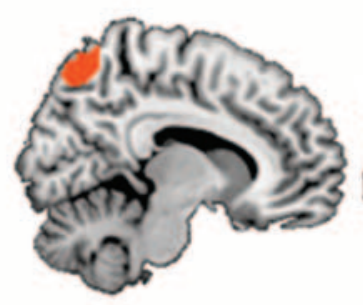

$x=9$

(c)

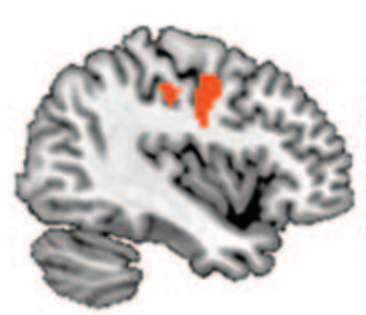

$x=-41$

(d)

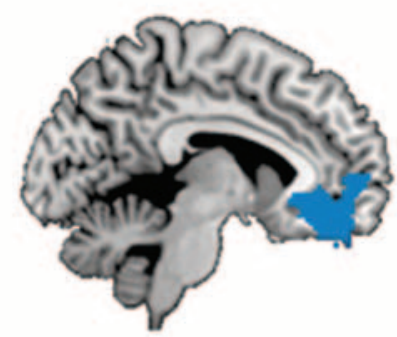

$x=-7$

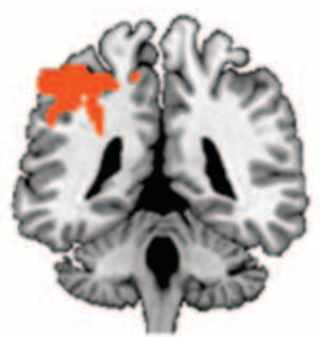

$y=-40$

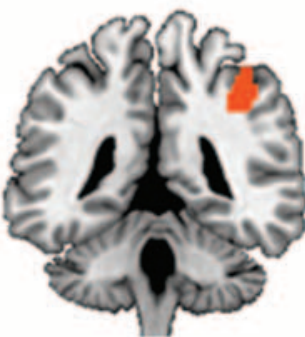

$y=-41$

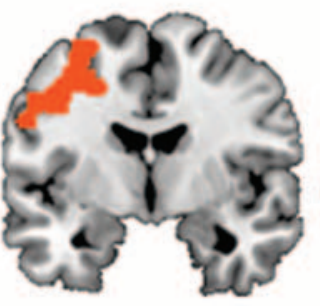

$y=-4$

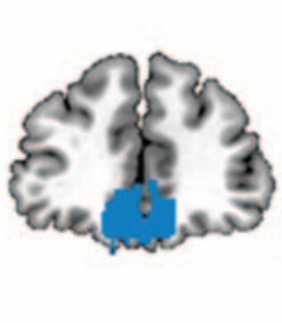

$y=41$

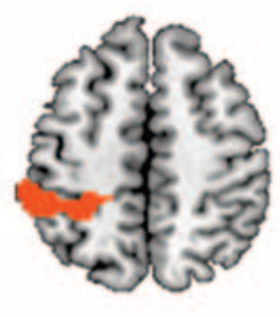

$Z=52$

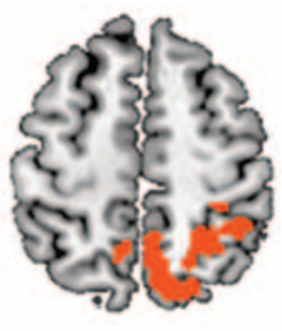

$Z=55$

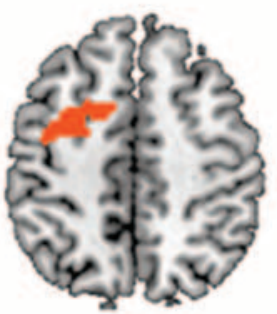

$Z=48$

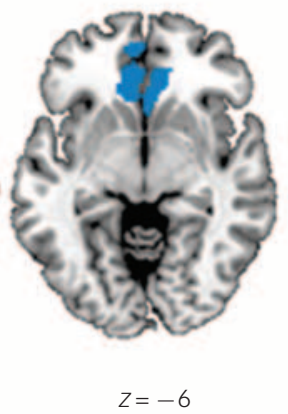

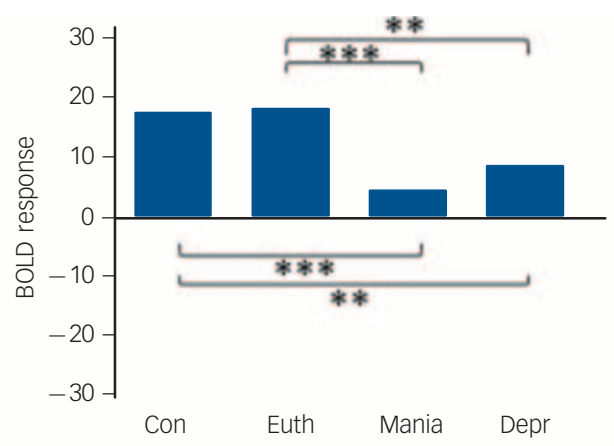

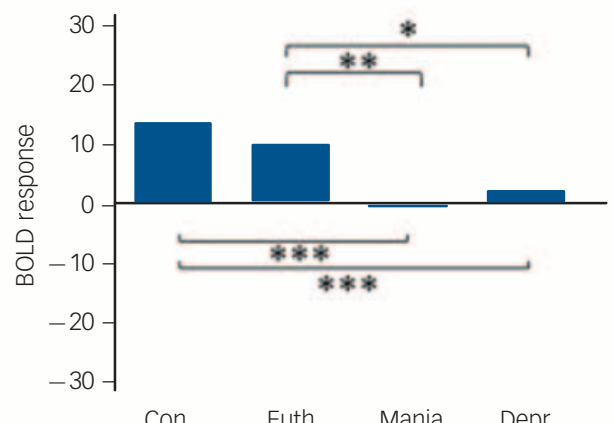

Con Euth Mania Depr
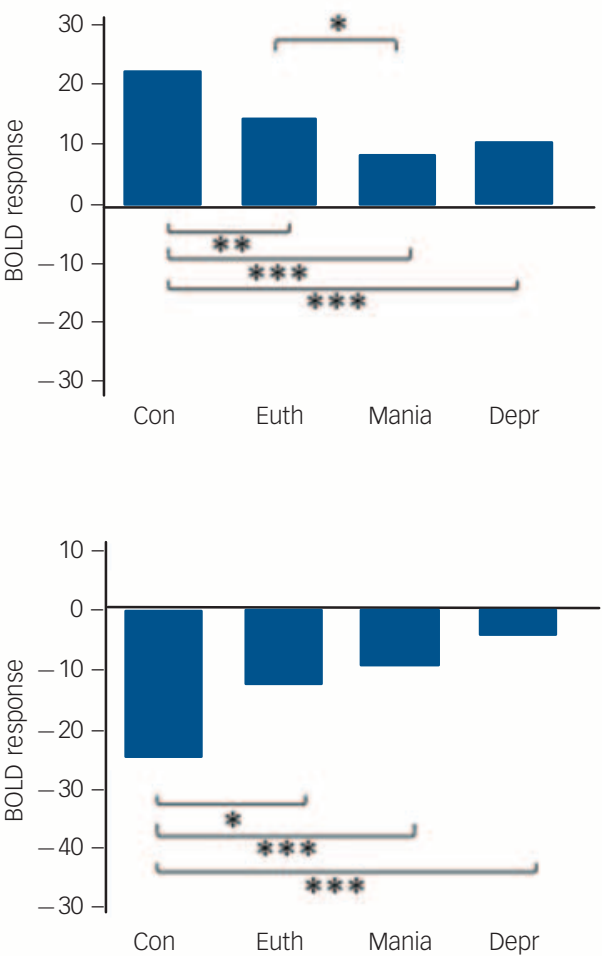

Fig. 4 The four clusters of significant difference found comparing all three bipolar subgroups and the control group.

The first three clusters, left parietal (a), right parietal (b) and left dorsolateral frontal (c) were regions of activation in the control group; the fourth in the ventromedial and orbitofrontal cortex (d) was a region where the control group showed de-activation. Boxplots are based on mean activation values from regions of interest (ROIs) extracted from the four significant clusters. BOLD, blood oxygen level-dependent; Con, control group; Euth, euthymia subgroup; Mania, mania subgroup; Depr, depression subgroup. ***significant at $P<0.001$, **significant at $P<0.01$, *all results significant at $P<0.05$ based on the post hoc Tukey's HSD test. The right side of the image is the right side of the brain.

Reduced activation in the dorsal parietal cortex was seen in both mania and depression but not in euthymia. Reduced activation was also seen in the dorsolateral prefrontal cortex, but here the changes showed a more complicated relationship with phase of illness - activation was reduced across all three phases of illness, although with greater reductions in mania (but not depression) than in euthymia. On the other hand, failure of de-activation in the medial frontal cortex was seen in all three illness phases and so seems to represent a trait-like abnormality. 


\section{Changes in the dorsal parietal and dorsolateral prefrontal cortex}

Our finding of mood-state dependent changes in the dorsal parietal cortex is unexpected - this region was not identified in either Kupferschmidt \& Zakzanis's ${ }^{5}$ or Chen et als ${ }^{6}$ meta-analyses, including in their subanalyses directed to mood state. One possible explanation for this relates to the fact that the parietal cortex forms part of the 'working memory network' activated the n-back task, ${ }^{28}$ and, although both meta-analyses pooled data from studies using a range of different cognitive tasks, relatively few of them employed the n-back or other working memory tasks (10/32 and 7/29 respectively). Among studies that did use the n-back task, Pomarol-Clotet et al ${ }^{10}$ found reduced activation in the parietal cortex along with other parts of the working memory network in patients in a manic phase, and FernándezCorcuera et $a l^{11}$ had similar results in bipolar depression (it should be noted that both these studies were carried out on samples that overlapped with the present study). With respect to euthymia, Cremaschi et $a l^{14}$ reviewed eight studies using the n-back task in this phase of illness and found reduced parietal activation only in one of them (Townsend et $a l^{7}$ - discussed further below); there no differences between patients and controls in three of the studies and three found increased activation. Accordingly, the literature to date provides some support for mood state-related differences in this region during performance of this cognitive task.

If this explanation is correct, it might be expected that a different pattern of mood-state dependent changes would be found if other tasks were used. Some support for this view comes from a study by Chen et al. ${ }^{29}$ They scanned 12 patients with bipolar disorder during performance of a facial emotion identification task, first when they were in a manic episode and then again when they had become euthymic. Twelve healthy controls were also scanned twice. A significant group $\times$ time interaction was found in the right amygdala and hippocampus, which was the result of increased activation in the patients when they were euthymic. (It should be noted that the authors used a mask restricting the analysis to brain regions involved in emotional processing and so the possibility of changes in other regions cannot be ruled out.)

We also found reduced activation in the dorsolateral prefrontal cortex, which showed ambiguous evidence of state-like characteristics. It did not appear in any of the contrasts between pairs of bipolar subgroups, but emerged in the ANOVA comparing all three bipolar subgroups and the controls. Reduced prefrontal cortex activation has been a regular finding in bipolar disorder, although it has mainly been documented in the orbitofrontal cortex ${ }^{30-32}$ the ventrolateral prefrontal cortex ${ }^{33}$ and the frontal pole ${ }^{34,35}$ perhaps reflecting the nature of the tasks used in these studies - the go/no-go task, the Stroop task and a gambling task. Consistent with such an interpretation, all of a small number of studies that have used working memory tasks have found reduced activation in or close to the dorsolateral prefrontal cortex in mania, ${ }^{10}$ depression ${ }^{11}$ and euthymia. ${ }^{36-38}$

The study of Townsend et al, ${ }^{7}$ described in the introduction, is the only other study to date that has examined patients in all three illness phases during performance of the n-back task. Their findings were quite different to ours in that they failed to find significant variation across phase in either the parietal cortex or the dorsolateral prefrontal cortex, although activation was reduced in all three patient groups compared with controls. There are two potential reasons why these authors may have failed to detect differences across phase, however. First, at 13-15 per group, the sample sizes may have been too small to detect differences, particularly when it is considered that between-patient differences are likely to be more subtle than those between patients and healthy controls. Second, group comparisons were carried out at the ROI rather than the whole brain level. Here it is noteworthy that, rather than using anatomically defined ROIs, the authors employed a $5 \mathrm{~mm}$ sphere around the maximally activated voxels in each bilateral region. It seems possible that this might have resulted in ROIs that favoured finding differences between patients and controls, but were not optimally located to detect differences between phases.

The activation changes between illness and euthymia we found in the dorsal parietal cortex, and more equivocally between mania and euthymia in the dorsolateral prefrontal cortex, could have reflected either the obvious symptomatic differences between the two states, or alternatively improvement in cognitive function taking place with recovery. Of the two, the latter seems intuitively more likely, given that we used a cognitive as opposed to an emotional task. A cautionary note needs to be sounded here, however, because the view that cognitive impairment in depression and mania normalises with clinical recovery ${ }^{39}$ is almost certainly an oversimplification. On the one hand, it is now accepted that cognitive impairment is also seen in a proportion of patients in euthymia (see, for example, Robinson \& Ferrier $^{40}$ ). On the other, the few studies that have directly compared the degree of impairment in different phases of illness have not found evidence that it is substantially less marked in euthymia (for example see Martinez-Aran et $\left.a l^{41}\right)$.

\section{Failure of de-activation in the medial frontal cortex}

The final finding of this study was that failure of de-activation in the medial frontal cortex distinguished the patients from the controls but was present to a similar degree in all three phases of illness. This finding is in line with those of our previous studies in mania ${ }^{10}$ and bipolar depression, ${ }^{11}$ although not with that of Strakowski et $\mathrm{al}^{12}$ in patients in a first-episode of mania. Calhoun et $a l^{42}$ additionally found failure of de-activation that affected particularly the medial frontal cortex in a mixed group of patients with bipolar disorder. Potentially also relevant here is a finding from Chen et al's ${ }^{6}$ meta-analysis of whole-brain voxel-based studies. This found the medial frontal cortex to be a site of increased activation compared with controls, and this continued to be seen in the subanalysis of the euthymia group. It is quite possible that this finding could actually represent failure of de-activation in the patients, since hyperactivation and failure of de-activation can give similar findings when conventional subtractive analysis is carried out (see Gusnard \& Raichle ${ }^{43}$ for a detailed explanation).

\section{Implications and limitations}

This study found that brain functional changes in bipolar disorder can be divided into those that are state-related and those that have more enduring, trait-like characteristics. The parietal cortex, an area that is implicated in working memory performance, showed evidence of belonging to the former category. Failure of de-activation in the medial frontal cortex, and so by implication default mode network dysfunction, appeared to fall into the latter. The dorsolateral prefrontal cortex showed a combination of both characteristics, exhibiting reduced activation in both phases of active illness but without fully normalising between episodes.

Our study has some limitations that should be acknowledged. Most notably, the patients were taking medication, which differed in dosage and type in the different phases of illness. We studied unselected patients with bipolar disorder: most but not all had type I illness, but we did not preselect patients with non-psychotic 
forms of illness. Additionally, the cross-sectional nature of the study means it may be susceptible to unknown, but potentially relevant, sampling biases.

Edith Pomarol-Clotet, MD, PhD, Silvia Alonso-Lana, BSC, FIDMAG, Germanes Hospitalàries, Barcelona and Centro de Investigación Biomédica en Red de Salud Mental (CIBERSAM), Spain; Noemi Moro, MD, FIDMAG, Germanes Hospitalàries and Benito Menni Complex Assistencial en Salut Mental, Barcelona, Spain; Salvador Sarró, MD, FIDMAG, Germanes Hospitalàries, Barcelona and Centro de Investigación Biomédica en Red de Salud Mental (CIBERSAM), Spain; Mar C. Bonnin, BSC, José M. Goikolea, MD, Centro de Investigación Biomédica en Red de Salud Menta (CIBERSAM) and Bipolar Disorder Program, Institute of Neuroscience, Hospital Clínic, University of Barcelona, IDIBAPS, Barcelona, Spain; Paloma Fernandez-Corcuera, $\mathrm{MD}$, Germanes Hospitalàries and Benito Menni Complex Assistencial en Salut Menta Barcelona, Spain; Benedikt L. Amann, MD, PhD, Anna Romaguera, MD, FIDMAG, Germanes Hospitalàries, Barcelona, Spain and Centro de Investigación Biomédica en Red de Salud Mental (CIBERSAM), Spain; Eduard Vieta, MD, PhD, Centro de Red de Salud Mental (CIBERSAM), Spain; Eduard Vieta, MD, PhD, Centro de
Investigación Biomédica en Red de Salud Mental (CIBERSAM) and Bipolar Disorder Program, Institute of Neuroscience, Hospital Clínic, University of Barcelona, IDIBAPS, Barcelona, Spain; Josep Blanch, MD, Hospital Sant Joan de Déu Infantil, Barcelona, Spain; Peter J. McKenna, MRCPsych, Raymond Salvador, PhD, FIDMAG, Germanes Hospitalàries, Barcelona, Spain and Centro de Investigación Biomédica en Red de Salud Mental (CIBERSAM), Spain

Correspondence: Edith Pomarol-Clotet, FIDMAG, Germanes Hospitalàries, Benito Menni CASM, C/. Dr. Antoni Pujadas 38, 08830 Sant Boi de Llobregat, Barcelona. Email: epomerol-clotet@fidmag.com

First received 28 Oct 2013, final revision 28 May 2014, accepted 6 Aug 2014

\section{Funding}

This work was supported by (a) the Centro de Investigación Biomédica en Red de Salud Mental (CIBERSAM); (b) several grants from the Instituto de Salud Carlos III (Miguel Servet Research Contract to E.P.-C. (CP10/00596), to B.A. (CP06/00359) and to R.S. (CP07/00048); intensification grant to S.S. (10/231) and to P.J.M. (12/325); PhD grant to S.A.-L. (FI11/00221); project grants to R.S. (PI05/1874 and PI10/01071); (c) the Comissionat per a Universitats Recerca del DIUE from the Catalonian Government (grants 2009 SGR211 and 2009 SGR1022).

\section{References}

1 Strakowski SM, Delbello MP, Adler CM. The functional neuroanatomy of bipolar disorder: a review of neuroimaging findings. Mol Psychiatry 2005; 10 105-16

2 Green MJ, Cahill CM, Malhi GS. The cognitive and neurophysiological basis of emotion dysregulation in bipolar disorder. J Affect Disord 2007; 103: 29-42.

3 Savitz J, Drevets WC. Bipolar and major depressive disorder: neuroimaging the developmental-degenerative divide. Neurosci Biobehav Rev 2009; 33 : 699-771.

4 Strakowski SM, Adler CM, Almeida J, Altshuler LL, Blumberg HP, Chang KD, et al. The functional neuroanatomy of bipolar disorder: a consensus model. Bipolar Disord 2012; 14: 313-25.

5 Kupferschmidt DA, Zakzanis KK. Toward a functional neuroanatomical signature of bipolar disorder: quantitative evidence from the neuroimaging literature. Psychiatry Res 2011; 193: 71-9.

6 Chen $\mathrm{CH}$, Suckling J, Lennox BR, Ooi C, Bullmore ET. A quantitative meta-analysis of fMRI studies in bipolar disorder. Bipolar Disord 2011; 13: 1-15.

7 Townsend J, Bookheimer SY, Foland-Ross LC, Sugar CA, Altshuler LL. fMRI abnormalities in dorsolateral prefrontal cortex during a working memory task in manic, euthymic and depressed bipolar subjects. Psychiatry Res 2010; 182: 22-9.

8 Raichle ME, MacLeod AM, Snyder AZ, Powers WJ, Gusnard DA, Shulman GL A default mode of brain function. Proc Natl Acad Sci U S A 2001; 98: 676-82.

9 Buckner RL, Andrews-Hanna JR, Schacter DL. The brain's default network: anatomy, function, and relevance to disease. Ann N Y Acad Sci 2008; 1124: $1-38$

10 Pomarol-Clotet E, Moro N, Sarro S, Goikolea JM, Vieta E, Amann B, et al. Failure of de-activation in the medial frontal cortex in mania: evidence for default mode network dysfunction in the disorder. World J Biol Psychiatry 2012; 13: 616-26.

11 Fernandez-Corcuera P, Salvador R, Monte GC, Salvador Sarró S, Goikolea JM Amann B, et al. Bipolar depressed patients show both failure to activate and failure to de-activate during performance of a working memory task. $J$ Affect Disord 2013; 148: 170-8.
12 Strakowski SM, Adler CM, Cerullo MA, Eliassen JC, Lamy M, Fleck DE, et al. MRI brain activation in first-episode bipolar mania during a response inhibition task. Early Interv Psychiatry 2008; 2: 225-33.

13 Allin MP, Marshall N, Schulze $\mathrm{K}$, Walshe $\mathrm{M}$, Hall MH, Picchioni $\mathrm{M}$, et al. A functional MRI study of verbal fluency in adults with bipolar disorder and their unaffected relatives. Psychol Med 2010; 40: 2025-35.

14 Cremaschi L, Penzo B, Palazzo M, Dobrea C, Cristoffanini M, Dell'Osso B, et al. Assessing working memory via N-back task in euthymic bipolar I disorder patients: a review of functional magnetic resonance imaging studies. Neuropsychobiology 2013; 68: 63-70.

15 Pomarol-Clotet E, Salvador R, Sarro S, Gomar J, Vila F, Martínez A, Guerrero A, et al. Failure to deactivate in the prefrontal cortex in schizophrenia: dysfunction of the default mode network? Psychol Med 2008; 38: 1185-93.

16 Salgado-Pineda P, Fakra E, Delaveau P, McKenna PJ, Pomarol-Clotet E Blin O. Correlated structural and functional brain abnormalities in the default mode network in schizophrenia patients. Schizophr Res 2011; 125: 101-9.

17 American Psychiatric Association. Diagnostic and Statistical Manual of Mental Disorder (4th edn) (DSM-IV). APA, 1994

18 Young RC, Biggs JT, Ziegler VE, Meyer DA. A rating scale for mania: reliability, validity and sensitivity. Br J Psychiatry 1978; 133: 429-35.

19 Hamilton, M. A rating scale for depression. J Neurol Neurosurg Psychiatry 1960; 23: 56-62.

20 Del Ser T, Gonzalez-Montalvo Jl, Martinez-Espinosa S, Delgado-Villapalos C Bermejo F. Estimation of premorbid intelligence in Spanish people with the Word Accentuation Test and its application to the diagnosis of dementia. Brain Cogn 1997; 33: 343-56.

21 Wechsler D. Wechsler Adult Intelligence Scale (3rd edn). Psychological Corporation, 1997

22 Gomar JJ, Ortiz-Gil J, McKenna PJ, Salvador R, Sans-Sansa B, Sarró S, et al. Validation of the Word Accentuation Test (TAP) as a means of estimating premorbid IQ in Spanish speakers. Schizophr Res 2011; 128: 175-6.

23 Gevins A, Cutillo B. Spatiotemporal dynamics of component processes in human working memory. Electroencephalogr Clin Neurophysiol 1993; 87: 128-43.

24 Green DM, Swets JA. Signal Detection Theory and Psychophysics. Krieger, 1966.

25 Smith SM, Jenkinson M, Woolrich MW, Beckmann CF, Behrens TE, Johansen-Berg $\mathrm{H}$, et al. Advances in functional and structural MR image analysis and implementation as FSL. Neuroimage 2004; 23 (suppl 1): S208-19.

26 GUy W. ECDEU Assessment Manual for Psychopharmacology. Revised DHEW Pub. (ADM). National Institute for Mental Health, 1976.

27 Jones SH, Thornicroft G, Coffey M, Dunn G. A brief mental health outcome scale - reliability and validity of the Global Assessment of Functioning (GAF). Br J Psychiatry 1995; 166: 654-9.

28 Owen AM, McMillan KM, Laird AR, Bullmore E. N-back working memory paradigm: a meta-analysis of normative functional neuroimaging studies. Hum Brain Mapp 2005; 25: 46-59.

29 Chen $\mathrm{CH}$, Suckling J, Ooi C, Jacob R, Lupson V, Bulmore ET, et al. A longitudinal fMRI study of the manic and euthymic states of bipolar disorder. Bipolar Disord 2010; 12: 344-7.

30 Blumberg HP, Leung HC, Skudlarski P, Lacadie CM, Fredericks CA, Harris BC, et al. A functional magnetic resonance imaging study of bipolar disorder: state- and trait-related dysfunction in ventral prefrontal cortices. Arch Gen Psychiatry 2003; 60: 601-9.

31 Elliott R, Ogilvie A, Rubinsztein JS, Calderon G, Dolan RJ, Sahakian BJ. Abnormal ventral frontal response during performance of an affective go/no go task in patients with mania. Biol Psychiatry 2004; 55: 1163-70.

32 Altshuler LL, Bookheimer SY, Townsend J, Proenza MA, Eisenberger N, Sabb $\mathrm{F}$, et al. Blunted activation in orbitofrontal cortex during mania: a functional magnetic resonance imaging study. Biol Psychiatry 2005; 58 763-9.

33 Mazzola-Pomietto P, Kaladjian A, Azorin JM, Anton JL, Jeanningros R. Bilateral decrease in ventrolateral prefrontal cortex activation during motor response inhibition in mania. J Psychiatr Res 2009: 43: 432-41.

34 Blumberg HP, Stern E, Ricketts S, Martinez D, de Asis J, White T, et al. Rostral and orbital prefrontal cortex dysfunction in the manic state of bipolar disorder. Am J Psychiatry 1999; 156: 1986-8.

35 Rubinsztein JS, Fletcher PC, Rogers RD, Ho LW, Aigbirhio Fl, Paykel ES, et al. Decision-making in mania: a PET study. Brain 2001; 124: 2550-63.

36 Monks PJ, Thompson JM, Bullmore ET, Suckling J, Brammer MJ, Williams SC, et al. A functional MRI study of working memory task in euthymic bipolar 
disorder: evidence for task-specific dysfunction. Bipolar Disord 2004; 6 : 550-64

37 Altshuler L, Bookheimer S, Townsend J, Proenza MA, Sabb F, Mintz J, et al Regional brain changes in bipolar I depression: a functional magnetic resonance imaging study. Bipolar Disord 2008; 10: 708-17.

38 Lagopoulos J, Ivanovski B, Malhi GS. An event-related functional MRI study of working memory in euthymic bipolar disorder. J Psychiatry Neurosci 2007; 32: 174-84.

39 Murray RM, Sham P, Van Os J, Zanelli J, Cannon M, McDonald C. A developmental model for similarities and dissimilarities between schizophrenia and bipolar disorder. Schizophr Res 2004; 71: 405-16.
40 Robinson L, Ferrier IN. Evolution of cognitive impairment in bipolar disorder: a systematic review of cross-sectional evidence. Bipolar Disord 2006; 8: 103-16.

41 Martinez-Aran A, Vieta E, Reinares M, Colom F, Torrent C, Sánchez-Moreno J, et al. Cognitive function across manic or hypomanic, depressed, and euthymic states in bipolar disorder. Am J Psychiatry 2004; 161: 262-70.

42 Calhoun VD, Maciejewski PK, Pearlson GD, Kiehl KA. Temporal lobe and "default" hemodynamic brain modes discriminate between schizophrenia and bipolar disorder. Hum Brain Mapp 2008; 29: 1265-75.

43 Gusnard DA, Raichle ME. Searching for a baseline: functional imaging and the resting human brain. Nat Rev Neurosci 2001; 2: 685-94.

\title{
On Memoirs of My Nervous IIIness, by Daniel Paul Schreber
}

\author{
Louis Sass
}

Daniel Paul Schreber's Memoirs of my Nervous IIIness profoundly influenced many key figures of modern psychiatry, including Bleuler and Jaspers, Freud and Jung. Author Elias Canetti described it as the 'most important document in psychiatric literature'. To read this work - typically considered a paradigmatic expression of paranoid schizophrenia - is to risk a shaking of one's complacency, especially concerning the key symptom of delusion. For me personally, it was a revelation.

Schreber was an appeals court judge and German citizen who wrote his memoir during an 8-year stay at the Sonnenstein hospital near Dresden. This dense, often convoluted work was published in 1903, together with documents from Schreber's successful suit for release from involuntary confinement. Against the asylum director's claim that the patient took his delusions and hallucinations for 'factual and real', with 'unshakeable certainty and [as] adequate motive for action', Schreber replied with 'the strongest possible "no" ': 'My Kingdom is not of this world', he wrote; those who think otherwise have 'not really entered into my inner spiritual life'.

Many of Schreber's reports are certainly bizarre: a foreign soul 'joined in looking out of my eyes'; 'the weather [was] dependent on my actions and thoughts'. He often claimed absolute certitude, and spoke of 'divine revelation ... founded on truth'. Yet it turns out to be difficult to assess the kind or quality of reality that Schreber either experienced or attributed to what he himself termed his 'so-called delusional system'. How, for example, should we understand his claim that people around him would temporarily exchange heads, or that his own internal organs were 'torn or vanished repeatedly', only to reappear 'without any permanent effect'? Was this simple irrationality and poor 'reality-testing', or does it suggest a less literal-minded sort of world - one schreber himself experienced as being more like a dream than a shared world of real consequences?

Many of Schreber's (so-called) delusional experiences and claims do not, in fact, suggest the literalness and error so often assumed. He speaks, for example, of seeing 'rays' and other delusional entities 'only with my mind's eye'. Nor are the experiences well captured by the popular notion of an 'externalisation' bias. What Schreber describes often seems, in fact, less an objectifying of something inner or imaginary than a subjectivising, derealising, or internalising of something external and real. Thus, he often experienced the actual people or other creatures in the asylum as unreal: 'miracled up' or 'fleetingly improvised', and as existing only within range of his own gaze.

It is, perhaps, a certain solipsistic stance that is the most distinctive as well as 'psychotic' feature of the Memoirs. Indeed, Schreber sometimes suggests that he himself was the true centre of the world, not only because 'everything that happens is in reference to me', but in the deeper, metaphysical sense that things only existed within his own point of view. 'Seeing' itself, says schreber, is 'confined to my person and immediate surroundings'.

All this is exceptionally hard to sort out in terms of any standard notions of knowledge, belief, and error - Schreber himself speaks of 'a tangle of contradictions that cannot be unraveled'. The abnormality seems to involve not mere error or cognitive bias, but an entirely different constitution of the world itself. Grasping the possibility of such overall, ontological alterations would seem crucial for any psychotherapist concerned about the patient's viewpoint; but also for neurobiologists and cognitive scientists: both the experience and underlying 'mechanisms' of at least some delusions can, it seems, be vastly different from what we typically imagine. schreber's memoir is a book to be sampled, savoured, and pondered, especially for the challenges it poses to standard assumptions, whether from common sense, philosophy, or psychiatric theory.

Daniel Paul Schreber. Memoirs of my Nervous Illness. Transl \& ed Ida Macalpine, Richard A. Hunter. Cambridge MA: Harvard University Press, 1988 (Orig: Denkwürdigkeiten eines Nervenkranken. Leipzig 1903). Quotations, listed in the order of appearance, on pp. 320, 301f, 157, 47, 41, 207, 301, 134, 99, 227, 101-107, 197, 232, 152. 